\title{
Superior Regularity in Erosion Patterns by Planar Subsurface Channeling
}

\author{
Alex Redinger, ${ }^{1}$ Henri Hansen, ${ }^{1}$ Udo Linke, ${ }^{2}$ Yudi Rosandi, ${ }^{3}$ Herbert M. Urbassek, ${ }^{3}$ and Thomas Michely ${ }^{1, *}$ \\ ${ }^{1}$ I. Physikalisches Institut, RWTH Aachen, 52056 Aachen, Germany \\ ${ }^{2}$ Institute of Thin Films and Interfaces, Forschungszentrum Jülich GmbH, 52425 Jülich, Germany \\ ${ }^{3}$ Fachbereich Physik, Universität Kaiserslautern, Erwin-Schrödinger-Straße, 67663 Kaiserslautern, Germany
}

(Received 9 November 2005; published 16 March 2006)

\begin{abstract}
The onset of pattern formation through exposure of $\mathrm{Pt}(111)$ with $5 \mathrm{keV} \mathrm{Ar}^{+}$ions at grazing incidence has been studied at $550 \mathrm{~K}$ by scanning tunneling microscopy and is supplemented by molecular-dynamics simulations of single ion impacts. A consistent description of pattern formation in terms of atomic scale mechanisms is given. Most surprisingly, pattern formation depends crucially on the angle of incidence of the ions. As soon as this angle allows subsurface channeling of the ions, pattern regularity and alignment with respect to the ion beam greatly improves. These effects are traced back to the positionally aligned formation of vacancy islands through the damage created by the ions at dechanneling locations.
\end{abstract}

DOI: 10.1103/PhysRevLett.96.106103

By grazing incidence ion scattering from metal surfaces a wealth of information and a deep understanding of many aspects of the ion-surface interaction has been obtained. Charge exchange between surface and projectile, electronic energy loss of the projectile, as well as electron emission due to scattering were extensively investigated (for a current review see [1]). The grazing incidence scattering geometry also played an important role in uncovering the interaction of highly charged ions with surfaces [24]. Despite this impressive knowledge gathered primarily through the analysis and spectroscopy of the scattered particles, very little is known about the damage left behind on the surface as a consequence of the collision. This is more surprising, as this damage is relevant whenever the scattered ions are used as a measuring tool for surface processes or structure (e.g., [5]). It is even crucial in applications of grazing ion beams like ion beam smoothening [6], thin film growth manipulation [7], nanopattern formation [8,9], or ion beam milling [10].

One step towards an interpretation of morphologies resulting from grazing incidence ion bombardment is the distinction between terrace damage and step edge damage [11]. Here we show for the first time how a specific damage mechanism at step edges acting only under grazing incidence conditions couples into the formation of mesoscopic damage structures. The alignment and overall periodicity of erosion patterns improve dramatically if the ion beam is tuned to conditions where planar subsurface channeling takes place. Planar subsurface channeling is the guided motion of ions between lattice planes parallel to the surface. It was discovered in ion-surface scattering experiments by characteristic energy losses of the scattered particles [12].

The experiments were performed in an ultrahigh vacuum variable temperature scanning tunneling microscopy (STM) apparatus with a base pressure in the $10^{-11}$ mbar range. Sample cleaning was accomplished by flash annealing to $1273 \mathrm{~K}$ and sputtering by a mass separated ion beam. For the grazing incidence experiments the clean surface
PACS numbers: 81.16.Rf, 61.80.Jh, 79.20.Ap, 79.20.Rf

was exposed to fluxes in the range of $1.8-2.8 \times$

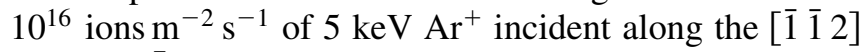
and the [110] direction at angles $\vartheta \in\left[78^{\circ}, 89^{\circ}\right]$ to the surface normal at various exposure times and temperatures. In the following the ion fluence $F$ (the product of ion flux and exposure time) is specified in monolayer equivalents $(\mathrm{MLE})$, where $1 \mathrm{MLE}=1.504 \times 10^{19}$ ions $/ \mathrm{m}^{2}$. Here we focus on the onset of pattern formation $(F \leq 2 \mathrm{MLE}$, ion beam in the [ $\left[\begin{array}{lll}\overline{1} & \overline{1} & 2\end{array}\right]$ direction) between 350 and $550 \mathrm{~K}$.

In order to elucidate the atomistic details of the processes occurring under ion impact, we performed molecular-dynamics (MD) simulations of these events. Simulations were performed for a target crystallite both at 0 and at $550 \mathrm{~K}$. While the simulations at $0 \mathrm{~K}$ are standard [13], we note that for the high-temperature simulations it was essential to fix the bottommost layer of the crystallite in order to stabilize it against "floppy" long-wavelength vibrational and torsional modes.

Figure 1 compares STM topographs after ion bombardment with $\vartheta=79^{\circ}$ [Figs. 1(a)-1(c)] and $\vartheta=83^{\circ}$ [Figs. 1(d)-1(f)] at $550 \mathrm{~K}$. The ion fluences increase from top to bottom and were chosen such that the eroded amounts for the two sequences are comparable. The direction of the ion beam is indicated in Fig. 1(a) by a white arrow. We first consider the case of $\vartheta=79^{\circ}$. After 0.25 MLE in Fig. 1(a) a large number of compact vacancy islands of monolayer depth with step edges aligned along the dense packed $\langle 110\rangle$ directions are present. Also few small adatom islands are visible. According to our MD simulations at $\vartheta=79^{\circ}$ ion impacts on a perfect terrace cause only small sputtering and adatom production (terrace sputtering yield $Y_{\mathrm{S}}^{\text {terr }} \approx 0.44$ and terrace adatom yield $Y_{\mathrm{A}}^{\text {terr }} \approx 2.7$ ), while impacts hitting an ascending step edge either directly or after reflection from the terrace cause significant sputtering (step edge sputtering yield $Y_{\mathrm{S}}^{\text {step }} \approx$ 9.9 and adatom yield $Y_{\mathrm{A}}^{\text {step }} \approx 28$ ). Neglecting preexisting steps, initially only terrace impacts take place. Only few surface vacancies are formed which agglomerate to va- 

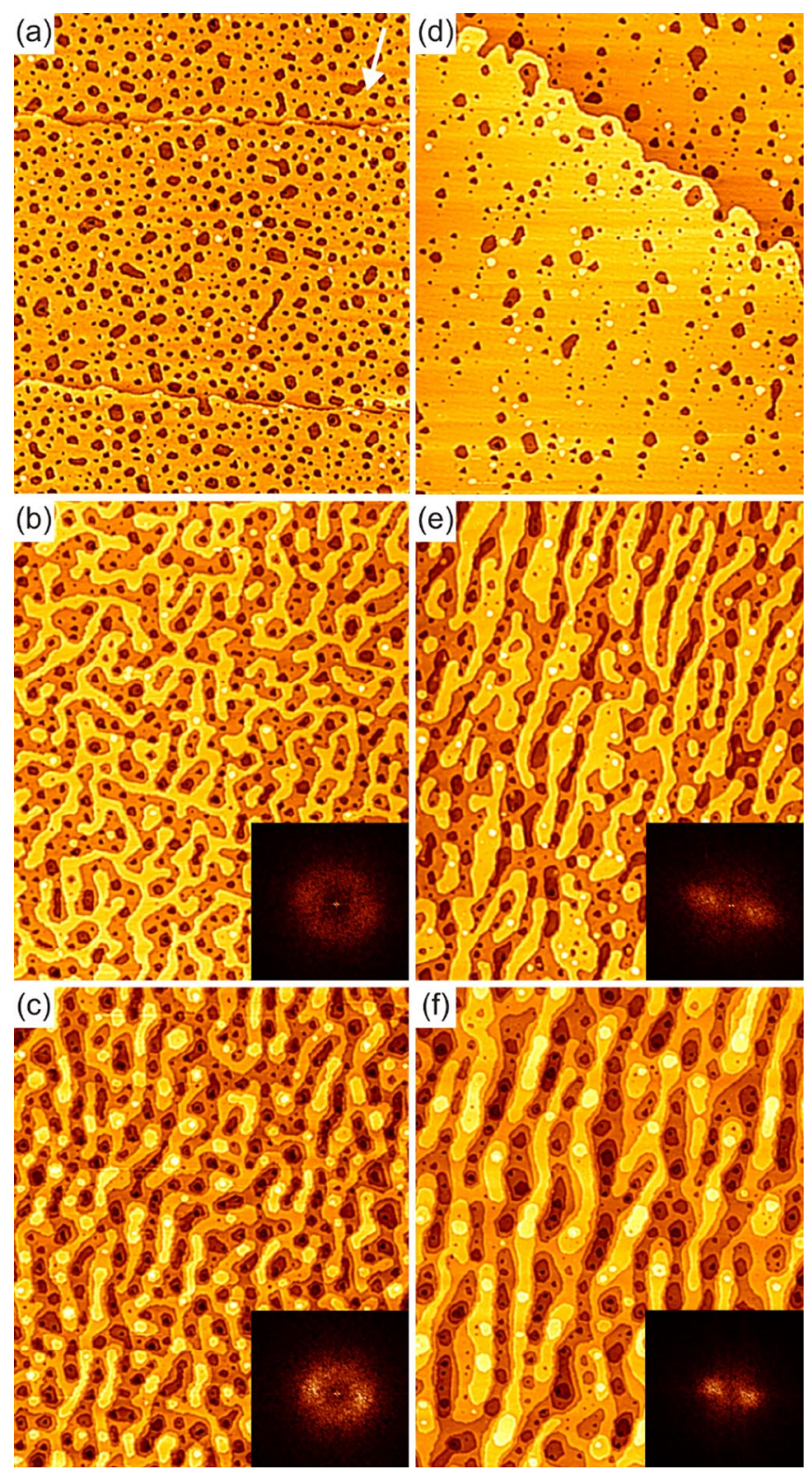

FIG. 1 (color online). STM topographs of Pt(111) after exposure to (a) 0.25 MLE, (b) 0.5 MLE, (c) 1.0 MLE, (d) 0.5 MLE, (e) 1.0 MLE and (f) $2.0 \mathrm{MLE}$ of $5 \mathrm{keV} \mathrm{Ar}^{+}$at $550 \mathrm{~K}$. The ion beam has an angle of incidence with respect to the surface normal of (a)-(c) $\vartheta=79^{\circ}$ and (d)-(f) $\vartheta=83^{\circ}$. The ion exposures were chosen to yield similar eroded amounts in each row. The direction of the ion beam is indicated by a white arrow in (a). All steps visible are of monolayer height. The image size is always $2200 \AA \times 1800 \AA$. Insets: power spectral densities of the topographs shown.

cancy clusters. After a certain induction time these clusters reach a size such that ions are able to hit the atoms of their illuminated step edge. Large angle scattering events with significant sputtering thus take place and the clusters start to grow rapidly to stable vacancy islands. The growing vacancy islands remain compact, as at $550 \mathrm{~K}$ step edge diffusion is sufficiently activated to keep the island shape close to its minimum energy shape composed predomi- nantly from $\langle 110\rangle$-oriented steps [14]. Upon increase of the ion fluence the randomly distributed compact vacancy islands coalesce and a labyrinth pattern of vacancy island grooves separated by remainder walls of the top terrace results [Fig. 1(b)]. The resulting long and narrow vacancy grooves are not transformed into compact islands as their size (step edge length) increases suddenly upon coalescence, making reshaping of the grooves slow on the experimental time scale [15]. Moreover, due to step-step repulsion [17], the grooves and the top terrace walls are hindered to reshape by newly formed lower layer compact vacancy islands. It is also apparent in Fig. 1(b) that the compact vacancy islands are formed with much higher probability at the bottom of the vacancy grooves rather than on the separating walls. The reason is simply that vacancies created by terrace impacts in the walls rapidly anneal to adjacent steps in the same layer, whereas vacancies created in the bottom of grooves are hindered to anneal to ascending steps by the step edge barrier for vacancies [14]. The transition to a multilayer pattern of ridges and grooves [compare Fig. 1(c)] is driven by this diffusion bias for vacancies. While the pattern of Fig. 1(b) still looks isotropic, in Fig. 1(c) some alignment of the resulting pattern with respect to the ion beam is present (compare also insets). It can be best identified if one focuses on the wall remainders of the upper layers: if not compact they are all more less oriented along the beam direction. The reason for this effect is twofold: step edge diffusion is ineffective for reshaping due to the reasons mentioned above (length scale, step-step repulsion), and the ion beam causes a global motion of all illuminated steps in beam direction due to sputtering. Thus the parts of the wall pattern in Fig. 1(b) normal to the beam will be removed first (small thickness normal to the beam) while the parts in the beam direction survive longer (large thickness normal to the beam) thereby leading to the aligned remainders of Fig. 1(c).

The morphological evolution is quite different for $\vartheta=$ $83^{\circ}$ as shown in Figs. 1(d)-1(f). Due to the increase of $\vartheta$ the ion kinetic energy in the direction normal to the surface decreases significantly. Damage on the terrace hardly takes place $\left(Y_{\mathrm{A}}^{\text {terr }} \leq 0.5\right.$ according to the MD simulations), whereas the step edge damage remains in magnitude similar to the values for $\vartheta=79^{\circ}$. For $\vartheta=83^{\circ}$ the yields $Y_{\mathrm{S}}^{\text {terr }}$ and $Y_{\mathrm{S}}^{\text {step }}$ were determined experimentally $\left(Y_{\mathrm{S}}^{\text {terr }}=0.08\right.$ and $\left.Y_{\mathrm{S}}^{\text {step }}=8.4\right)$ and agree quantitatively with the yields obtained from MD simulations $\left(Y_{\mathrm{S}}^{\text {terr }}=0\right.$ and $Y_{\mathrm{S}}^{\text {step }}=8.3$ ) $[11,18]$. Because of the reduced terrace damage the initial induction phase is extended, and roughly a twice as large ion fluence $(0.5 \mathrm{MLE})$ is needed to reach a similar eroded amount in Fig. 1(d) compared to Fig. 1(a). The more difficult initial vacancy cluster formation also causes a reduction in the number density of vacancy islands in Fig. 1(d) compared to Fig. 1(a). Viewing Figs. 1(a) and 1(d) under grazing angles from various directions reveals another striking difference of the two morphologies: 
Contrary to Fig. 1(a), in Fig. 1(d) a considerable amount of positional order of the vacancy islands is visible. The islands tend to form chains aligned along the ion beam direction. This aligned formation of vacancy islands causes, during their subsequent growth, preferential coalescence in ion beam direction. As a result long and narrow vacancy grooves are formed, which are well aligned in the ion beam direction as apparent in Fig. 1(e). The alignment in Fig. 1(e) is obvious if opposed to the isotropic situation for $\vartheta=79^{\circ}$ in Fig. 1(b) after a similar removed amount. The insets in Fig. 1 exhibiting the power spectral densities highlight the qualitatively different ordering behavior. The ratios of the amplitudes of the dominant wavelengths in the morphology normal and parallel to the ion beam are 1.3 for Fig. 1(b) and 3.8 for Fig. 1(e). As for the $\vartheta=79^{\circ}$ case, the situation at coalescence [Fig. 1(e)] transforms upon further exposure into a multilayer pattern of grooves and ripples, which has, however, a much better alignment with respect to the ion beam and a better defined periodicity [Fig. 1(f)]. The initial difference in ordering and periodicity for the two bombardment geometries remains pronounced even for large ion fluences of 20 MLE (topographs not shown). Finally, the same marked dependence in the alignment of the vacancy islands and pattern regularity on $\vartheta$ is found for the ion beam incident along the [110] direction. Details will be given elsewhere.

The greatly improved ordering and pattern alignment for $\vartheta=83^{\circ}$ has to be traced back to the positional alignment of vacancy islands giving the impression of island chains in Fig. 1(d). What is the origin of this aligned island formation? To this end we performed MD simulations for $\vartheta=$ $83^{\circ}$ at $550 \mathrm{~K}$ and various impact locations near the step edge. If the ion hits the step edge close to its bottom, we find a significant chance that the ion enters the space between the top layer and the layer below. In this space it performs an oscillatory motion guided by the (for the fast moving ion) nearly planar repulsive potentials of the two layers. Where the ion hits the surface layer from below a few atoms are pushed onto the surface layer resulting in adatoms. While the oscillation period at $550 \mathrm{~K}$ is not well defined due to the influence of thermal vibrations on the ion trajectory, our $0 \mathrm{~K}$ simulations exhibit an oscillation period of about $23 \AA$, resulting in regularly spaced damage spots with about 4 adatoms on average. Eventually, due to loss of energy and a large angle scattering event, the ion dechannels. In the example of Fig. 2(a) this takes place as much as $110 \AA$ behind the step. A considerable amount of damage is created at the location of dechanneling: an adatom cluster containing 19 adatoms and an even larger surface vacancy cluster are visible. Single defects and small clusters of adatoms and vacancies created along the channeling path are mobile at $550 \mathrm{~K}$ and, consequently, there is a significant chance to heal these defects (either by recombination or diffusion to sinks) prior to subsequent ion impacts. In contrast, the large clusters caused by dechanneling are likely to survive immediate healing. Subsequent

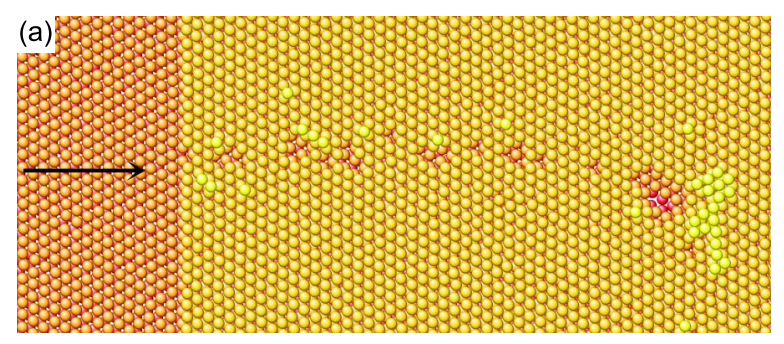

(b)

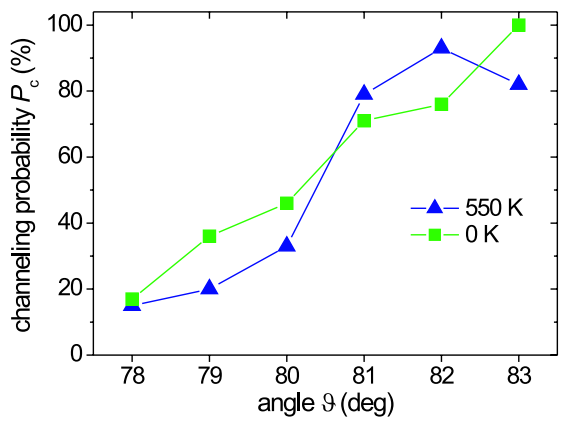

FIG. 2 (color online). (a) MD simulation damage pattern of a $5 \mathrm{keV} \mathrm{Ar}{ }^{+}$ion incident on $\operatorname{Pt}(111)$ at $550 \mathrm{~K}$ with $\vartheta=83^{\circ}$. The ion enters the space between the upper terrace and the layer underneath at the step edge and performs planar subsurface channeling (see text). (b) Channeling probability $P_{\mathrm{c}}$ for $5 \mathrm{keV}$ $\mathrm{Ar}^{+}$ions hitting a dense packed $\mathrm{Pt}(111)$ step edge at the location of largest $P_{\mathrm{c}}$ in dependence of the angle of incidence $\vartheta$. Molecular-dynamics results for $0 \mathrm{~K}$ (solid squares) and $550 \mathrm{~K}$ (solid triangles).

step edge impacts into the cluster steps will cause the formation of a new independent vacancy island. Assuming the step, where the channeling ion initially entered, to be the ascending boundary of a mother vacancy island, this process causes the formation of a daughter vacancy island. With respect to the ion beam direction, the daughter vacancy island is found behind the mother vacancy island due to its origin from the dechanneling damage. By iteration, this mechanism leads to chains of vacancy islands aligned in the ion beam direction as visible in Fig. 1(d). Although vacancy island formation is too rare to allow efficient pattern formation for ion bombardment with $\vartheta=86^{\circ}$, experiments for this polar angle corroborate our picture. In these experiments the probability of observing a daughter island behind a mother island sharply increases from values close to zero to values near 1 as soon as the mother island diameter exceeds $35 \AA$, i.e., as soon as the lateral mother island extension allows impinging ions to impact at the bottom of the illuminated mother island step and to perform subsurface channeling.

If the vacancy island chain formation and the pattern alignment along the beam direction is in fact due to the subsurface channeling, from the morphologies of Fig. 1 we have to conclude that subsurface channeling is largely diminished for $\vartheta=79^{\circ}$. Using MD simulations we analyzed the subsurface channeling probability $P_{\mathrm{c}}$ of $5 \mathrm{keV}$ $\mathrm{Ar}^{+}$ions in dependence of $\vartheta$. An ion is considered as a channeling ion if it stayed at least for the $0 \mathrm{~K}$ oscillation 

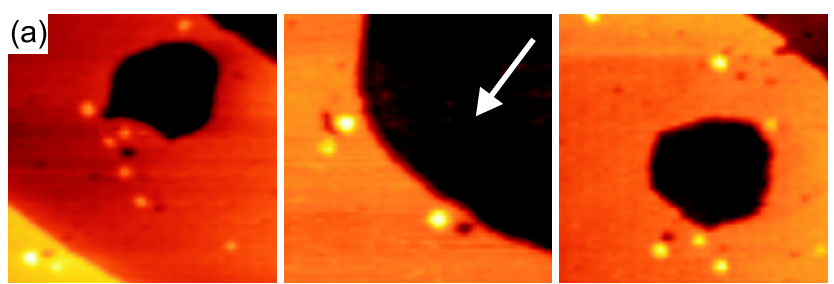

(b)

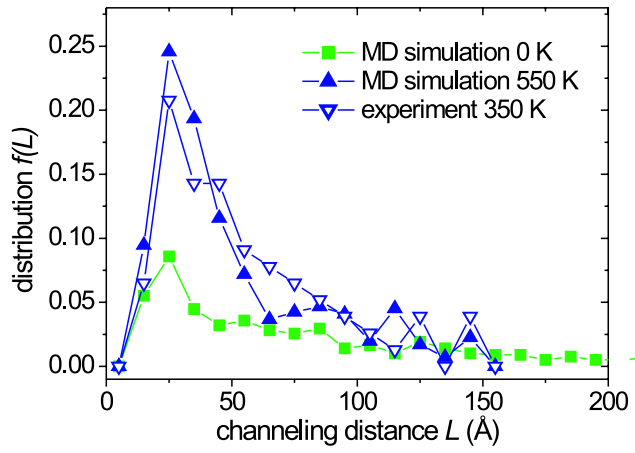

FIG. 3 (color online). (a) STM topographs after exposing $\mathrm{Pt}(111)$ with prefabricated steps (large equilibrium shaped vacancy islands) to an extremely low fluence of $5 \mathrm{keV} \mathrm{Ar}^{+}$ions at $\vartheta=83^{\circ}$ and $350 \mathrm{~K}$. Damage patterns of single ion impacts are visible at the illuminated steps (see text). The ion beam direction is indicated by a white arrow. The image size is always $310 \AA \times$ $310 \AA$ A. (b) Distribution of channeling distances $L$ for $5 \mathrm{keV} \mathrm{Ar}{ }^{+}$ ions at $\vartheta=83^{\circ}$ incidence. Solid squares: MD simulation at $0 \mathrm{~K}$; solid triangles: MD simulation at $550 \mathrm{~K}$; open triangles: experiment at $350 \mathrm{~K}$. Data normalized to $\int f(L) d L=1 ; 54 \%$ of the $0 \mathrm{~K}$ data have channeling lengths outside the range shown here.

wavelength of $23 \AA$ between the two lattice planes. $P_{\mathrm{c}}$ is determined for the impact location, where channeling is most likely [19]. As shown in Fig. 2(b), $P_{\mathrm{c}}$ dramatically increases at $550 \mathrm{~K}$ from $20 \%$ for $\vartheta=79^{\circ}$ to more than $80 \%$ for $\vartheta=83^{\circ}$, in full agreement with our hypothesis.

Additional evidence for the presence of planar subsurface channeling comes from STM topographs. Figure 3(a) exhibits STM topographs after exposing prefabricated steps to the low ion fluence of $0.001 \mathrm{MLE}$ at $350 \mathrm{~K}$ and subsequent room temperature imaging. The defect patterns at the step are due to single step edge impacts. Assuming the centers of the vacancy clusters behind the illuminated step edges to mark the locations of dechanneling, we were able to obtain an experimental channeling distance distribution as shown by open triangles in Fig. 3(b). It is compared to the channeling distance distributions obtained by MD simulations at $0 \mathrm{~K}$ [solid squares in Fig. 3(b)] and $550 \mathrm{~K}$ [solid triangles in Fig. 3(b)]. The long tail in the channeling distance distribution at $0 \mathrm{~K}$ is due to the absence of thermal vibrations and vanishes already at room temperature. The good agreement between the experimental 350 and the $550 \mathrm{~K}$ MD simulation distribution lends additional credit to our interpretation. Imaging of single ion impact patterns at low temperatures is certainly necessary to make more detailed statements.
In conclusion, in the absence of subsurface channeling, pattern alignment relies on the preferential removal of step edges illuminated by the ion beam. The alignment starts with remainders in upper layers, which are fixed by stepstep repulsion and which are too large to reshape efficiently by step edge diffusion. However, if the angle of incidence in ion bombardment allows subsurface channeling with significant probability, pattern alignment and regularity greatly improve due to the aligned formation and coalescence of vacancy islands as a consequence of the dechanneling damage of single ions. The discovery of the link between subsurface channeling and patterns with superior alignment and regularity is of importance for future nanoscale pattern fabrication experiments. It will allow the use of MD simulations as a guide to optimum channeling conditions and thus to highly perfect patterns.

We acknowledge useful discussions with Celia Polop, Georgiana Stoian, and Joachim Krug. This work was supported by the Deutsche Forschungsgemeinschaft via the project "Streifender Ionenbeschuss" and by TPSDP Ministery of National Education, Republic of Indonesia.

*Electronic address: michely@physik.rwth-aachen.de

[1] H. Winter, Phys. Rep. 367, 387 (2002).

[2] A. Arnau et al., Surf. Sci. Rep. 27, 113 (1997).

[3] C. Lemell, J. Stöckl, J. Burgdörfer, G. Betz, H. P. Winter, and F. Aumayr, Phys. Rev. Lett. 81, 1965 (1998).

[4] A. Robin, D. Niemann, N. Stolterfoht, and W. Heiland, Phys. Rev. A 67, 052901 (2003).

[5] R. Pfandzelter, T. Bernhard, and H. Winter, Phys. Rev. Lett. 90, 036102 (2003).

[6] M. Wißing, M. Batzill, and K. J. Snowdon, Nanotechnology 8, 40 (1997).

[7] K. C. Ruthe and S. A. Barnett, Surf. Sci. 538, L460 (2003).

[8] S. van Dijken, D. de Bruin, and B. Poelsema, Phys. Rev. Lett. 86, 4608 (2001).

[9] M. Ovsyanko, G. Stoian, H. Wormeester, and B. Poelsema, Phys. Rev. Lett. 93, 086103 (2004).

[10] B. W. Kempshall, S. M. Schwarz, B. I. Prenitzer, L. A. Gianuzzi, R. B. Irwin, and F.A. Stovie, J. Vac. Sci. Technol. B 19, 749 (2001).

[11] H. Hansen et al., Phys. Rev. Lett. 92, 246106 (2004).

[12] K. Kimura, M. Hasegawa, and M. H. Mannami, Phys. Rev. B 36, 7 (1987).

[13] A. Friedrich and H. M. Urbassek, Surf. Sci. 547, 315 (2003).

[14] T. Michely et al., Phys. Rev. Lett. 86, 2589 (2001).

[15] The reshaping time $\tau$ of a deformed island increases as $\tau \propto d^{\alpha}$, where $d$ is the final, compact island diameter and $\alpha$ ranges between 3 and 4 [16].

[16] M. Eßer, K. Morgenstern, G. Rosenfeld, and G. Comsa, Surf. Sci. 402, 341 (1998).

[17] E. Hahn, H. J. Schief, V. Marsico, A. Fricke, and K. Kern, Phys. Rev. Lett. 72, 3378 (1994).

[18] With $Y_{\mathrm{S}}^{\text {terr }}, Y_{\mathrm{S}}^{\text {step }}$ and the time dependent concentration of illuminated steps the experimental erosion rate is quantitatively reproduced for $\vartheta=83^{\circ}$.

[19] The location was at $0.5 x_{c}$ in the terminology of Ref. [11]. 